\title{
Limitations of anthropic predictions for the cosmological constant $\Lambda$ : cosmic heat death of anthropic observers
}

\author{
Laura Mersini-Houghton ${ }^{1}$ and Fred C Adams ${ }^{2}$ \\ ${ }^{1}$ Department of Physics and Astronomy, UNC-Chapel Hill, NC 27599-3255, USA \\ 2 Michigan Center for Theoretical Physics, University of Michigan, Ann Arbor, MI 48109, USA \\ E-mail: mersini@physics.unc.edu and fca@umich.edu
}

Received 16 April 2008, in final form 27 May 2008

Published 30 July 2008

Online at stacks.iop.org/CQG/25/165002

\begin{abstract}
This paper investigates anthropic bounds on the vacuum energy $\Lambda$ by considering alternate starting assumptions. We first consider the possibility of cosmic observers existing at any random time (including the future) for constant $\Lambda$, and take into account the suppression of new structure formation as the universe approaches its eternal de Sitter (DS) geometry. Structures that collapse prior to the era of $\Lambda$-domination will lose causal contact with our Hubble volume within a finite (short) conformal time $\tau_{*}$. Any remnants within our Hubble volume then suffer a cosmological heat death after the universe becomes DS. The probability for finding observers by random measurements in the volume bound by the de Sitter horizon is proportional to the rate of change in the ratio of the 3 -volume $V_{3}(\tau)$ to the 4 -volume $V_{4}(\tau)$, so that $P \simeq 0$. This vanishing probability of populated DS volumes is a simple consequence of the information loss problem for eternal DS spaces resulting from the finite and constant value of its temperature $T_{D S} \simeq \Lambda^{-1 / 2}$ and entropy $S=3 /(G \Lambda)$. By contrast, for geometries with $\Lambda=0$, structures can condense and entropy production can continue without bounds at any epoch. The probability of finding observers in $\Lambda=0$ geometries is thus overwhelming higher than in DS spaces. As a result, anthropic reasoning does not explain the small but nonzero vacuum energy observed in our universe. We also address the case where observers are considered only at a specially chosen time-like the present epoch-but relax the allowed values of starting density fluctuations and hence the redshift of galaxy formation. In this latter case, the bounds on a $\Lambda$ can be millions of times larger than previous estimates-and the observed value. We thus conclude that anthropic reasoning has limited predictive power.
\end{abstract}

PACS number: 98.80.Qc 


\section{Introduction}

This paper investigates the implications of anthropic reasoning on the selection of the level of vacuum energy $\Lambda$. Our universe is currently observed to be in an accelerated expansion [1], although the nature and origin of the dark energy that propels the expansion of the universe into an accelerated phase remains a mystery. The simplest manifestation of the dark energy would be a pure cosmological constant $\Lambda$, where $\Lambda \approx 10^{-122} M_{p}^{4}$ for our universe at the present epoch; however, vacuum energy that varies with time is also possible.

Both a pure cosmological constant $\Lambda$ and a dynamic mechanism for dark energy (so that the energy density of the vacuum can be time dependent) can give rise to similar Hubble expansions at present; as a result, current observations have difficulty distinguishing between them. However, the two models imply crucial differences in the future evolution of our Hubble volume. A constant vacuum energy density leads at the classical level [2] to an eternally DS space in thermal equilibrium, with an eternally finite and constant bound on the entropy given by the horizon size, $S_{D S}=3 / G \Lambda$. In contrast, a universe with dynamic dark energy has no bound on its growth of entropy.

Unlike the case of universes with dynamic dark energy components, a universe with a pure nonzero vacuum energy $\Lambda$ cannot produce new structures after it thermalizes at the DS temperature $T_{D S} \simeq \Lambda^{-1 / 2}$, since the bound given by $S_{D S}$ does not allow further entropy generation. In addition, any remnant condensates produced before thermalization at $T_{D S}$ disappear in the DS epoch since they either lose causal contact with our Hubble volume or suffer a cosmological heat death within the DS space. The DS volume empties out within a finite time of about $H^{-1}$. Any pre-existing astrophysical structures die out over a somewhat longer time interval. In the long term, when thermal equilibrium is reached, the temperature $T_{D S}$ and entropy $S_{D S}$ in this Hubble volume approach and then maintain a constant value at all times and all points in space; as a result, no further work can be done and no heat engine can operate. In the long term future, this space thus suffers from one type of cosmological heat death of its remnant structures [4].

One currently popular attempt at addressing the observed extraordinary small value of the vacuum energy $\Lambda$ is through anthropic selection. In this approach, the value of $\Lambda$ is treated as an environmental parameter that varies in different pocket universes or causally disconnected regions of the embedding background. The challenge for any anthropic selection argument is to first identify an observer and then estimate the probability distribution for measuring the observed parameters contained in each pocket universe. The hope is that the most typical value in this distribution for the $\Lambda$ parameter will turn out to be the one detected in our universe, thereby solving the naturalness issue for the observed vacuum energy $\Lambda$. As we discuss below, however, due to the eternal nature of the thermal equilibrium of DS spaces, the only allowed value for $\Lambda$ that would avoid the long-term extinction of observers by a cosmological heat death is the strict value $\Lambda=0$.

This paper is organized as follows. We first review, in section 2, the original anthropic argument [3] and several more recent generalizations. In section 3, we investigate anthropic arguments by considering the probability of finding observers randomly at any cosmological epoch and take into account their cosmological heat death; these considerations lead to the prediction of $\Lambda=0$. In section 4 , we present a second alternate argument that requires observers arise before the present epoch, but allows for earlier structure formation; these considerations allow for huge values of $\Lambda$. Note that the first of these arguments applies to constant $\Lambda$, whereas the second allows for dynamic dark energy. Finally, we conclude in section 5 with a summary of our results, a discussion of the various ways in which anthropic arguments can be constructed, and their limited predictive power. 


\section{Anthropic reasoning}

\subsection{Weinberg's proposal}

Well before the acceleration of the universe was discovered in 1998, in a seminal paper Weinberg [3] found an approximate upper bound on the vacuum energy, i.e., $\rho_{\Lambda} \leqslant 10^{3} \rho_{m}^{0}$, where $\rho_{m}^{0}$ is the present matter energy density; this bound arises because larger values of $\rho_{\Lambda}$ do not allow galaxies to form. Weinberg argued that since observers arise from non-equilibrium processes as the result of structure formation, then cosmological structures must condense (because observers are present). However, galaxy formation only takes place for sufficiently small energy densities of the vacuum, $\rho_{\Lambda} \leqslant \rho_{m}$, which is evaluated at the redshift $z_{*}$ when density perturbations become nonlinear. Relating this condition to present day values leads to the bound $\rho_{\Lambda} \leqslant\left(1+z_{*}\right)^{3} \rho_{0}$, where the subscript refers to the present epoch. Since $z_{*} \leqslant 10$, this argument leads to a bound on $\rho_{\Lambda}$ that is about 2-3 orders of magnitude larger than the observed value. Nonetheless, this bound sufficiently suppresses the value of $\Lambda$ compared to the 'standard' quantum gravity estimate of $M_{p}^{4}$, which is about 120 orders of magnitude too large. This improvement is considered by many to lend credence to the predictive powers of the anthropic reasoning.

It should be noted that Weinberg's argument selects a special time $z_{*}$ and was based on the criterion for the nucleation of condensates prior to $\Lambda$ domination. with no reference to the future evolution of these structures [19]. As such, the prior knowledge of the parameters $\left(\rho_{m}, z_{*}\right)$ can be considered as fine-tunings of the approach, as we explicitly explore in section 4. In addition, the argument remains (nearly) the same if the cosmological constant $\Lambda$ is replaced by some dynamic dark energy. Finally, we note that more recent work [12] updates the original Weinberg bound in light of the current observations of $\Lambda$, and reaches similar conclusions.

\subsection{Anthropic multiverse and the probability distribution of observers}

The recent observations of the accelerated expansion of the universe, which may be due to a pure vacuum energy $\Lambda$, in conjunction with the major theoretical challenges we face in explaining $\Lambda$, have revived interest in the anthropic reasoning as a possible means of predicting 'typical' values for the vacuum energy density. Of course, the hope is to 'predict' the observed value. Toward this end, various proposals for prescribing a probability distribution for pocket universes and their internal observers have been put forth (e.g., see [5]).

For these proposals, the general prescription is to express the probability $P_{j}$ for randomly finding an observer in pocket universe $j$ by the product of the concentration (priors) $p_{j}$ of $j$-type bubbles with a selection weight (conditional probability) $f_{j}$, i.e., $P_{j}=p_{j} f_{j}$. Here, $f_{j}$ is the weight assigned to the $j$ th bubble, where the weight is proportional to the average number of observers within the $j$ th bubble so that $f_{j} \propto N_{j}$.

One complication in this approach is related to the puzzle of Boltzmann brains (BBs) [20]. $\mathrm{BBs}$ arise from random quantum fluctuations about equilibrium and outnumber the regular observers (like us) that arise from non-equilibrium processes traced back to the nonlinear growth of density perturbation during the matter domination epoch, perturbations which themselves originate from the earliest times. If inflation is generically eternal, as believed till recently, then the number of pocket universes grows without bound. According to the Everett interpretation, the number of BBs and regular observers populating the continually produced bubbles will also grow without bound. As a result, using the ratio of Boltzmann brains to that of regular observers to estimate the probability distribution $P_{j}$ described above involves a ratio of infinities and thus becomes undefined without further specification. 
Recent attempts [7] propose to identify the production of Boltzmann brains with the bubble abundance factor $p_{j}$ rather than $f_{j}$ and identify observers in $f_{j}$ only as those arising from non-equilibrium fluctuations in order to regulate the nucleation rates of BBs and regular observers.

In the end, however, this anthropic pocket-based approach, like the original Weinberg argument, is concerned only with the nucleation rates of the observers (regular observers and $\mathrm{BBs}$ ), and does not include the decay rates in estimating the average number of observers $f_{j}$ for DS pocket-universes. As we argue below, the inevitable loss of observers in a DS spacetime changes the predictions from this line of reasoning.

For completeness we note that inflation may not be generically eternal, as shown recently by $[8,17]$. If this is the case, the puzzle of bubble population goes away. However, bubble production by tunneling or through the string theory landscape may lead to a multiverse picture similar to the one from eternal inflation.

\subsection{High complexity proposal}

Recently, the proposal of [9] uses similar reasoning to Weinberg's original selection criterion but replaces the prerequisite of galaxy formation for the nucleation of observers [3] by hypothesizing the requirement of a high entropy content $\Delta S$ within a causal patch as a sufficient complexity prerequisite to give rise to observers. The entropy production $\Delta S$ provides the weighting factor for the population term $f_{j}$ in the probability distribution described above using the conjectured principle that structure is most likely to be found in universes where the values $\Lambda$ allow the entropy inside a casually connected region to be maximized. Radiation from stellar burning, reprocessed by dust, is the major source of entropy in this case. Therefore the amount of entropy produced depends on the distribution of matter $M$ contained in halos by time $x=a^{3}$. The probability $F$ of a given volume being bound at scale factor $a$ is given by the Press-Schecter (PS) function [16], which can be written in the form

$$
F\left[\rho_{\Lambda}, M, x, x_{e}, \mu\right] \simeq \operatorname{Erfc}\left[\frac{A\left(\rho_{\Lambda} / \rho_{*}\right)}{G(x) s(\mu)}\right],
$$

where $A \simeq 5.6, \rho_{*}=\xi^{4} P^{3}, \mu=M \xi^{-2}$ and $\xi^{2}=M_{e}$. In addition, $G(x)$ is the growth function, $P$ is given by the power spectrum and $M_{e}$ is the mass contained within the volume at the time $x_{e}$ of matter-radiation equality. This function has a well-defined peak when its argument is near $10^{2} \rho_{*}$. Therefore $\mu$ expresses the mass at time $x$ relative to the horizon mass scale $\xi^{-2}$ at equality time $x_{e}$. The horizon mass scale at the equality time is the crucial physical scale built in the PS formula. The lack of the generation of new entropy when the universe has transited from the matter domination to the $\Lambda$ domination epoch, is given by the finite conformal time of the causal diamond $V_{3} \rightarrow 0$. Therefore the issue of a finite amount of entropy spread over an infinite range of physical time, described by the 4-volume $V_{4} \simeq H^{-2}$, or equivalently ignoring the influence of $\Lambda$ on these structures during the DS era, remains as severe in this proposal as in the previous ones. As an illustration of these effects, let us mention that photons from stellar burning are redshifted to infinity when these astrophysical objects are expelled from the causally connected region. In this sense both arguments [3, 9] are similar. The improvement in the value of dark energy density [9], compared to the value found in [3] comes from requiring that the maximum entropy estimated with the PS formula be near the maximum volume of the causal diamond.

Indirectly, this argument contains a fine-tuning of the dark energy coincidence puzzle. The $\mu$ scale of the PS function introduces the condition that the matter-dark energy equality time $x_{\Lambda}$ occurs close to the time of matter-radiation equality $x_{e}$. Requiring that the coincidence 
puzzle for $\Lambda$ be satisfied at a special time, namely when $\rho_{\Lambda} \simeq \rho_{m}$ near present time, leads to an improved value for $\Lambda$ compared to the one in [3].

\section{Cosmological heat death of anthropic observers}

In this section we revisit the limits that anthropic considerations place on the cosmological constant $\Lambda$. In a $\Lambda$ CDM universe that classically becomes an eternal de Sitter space, the time during which structure and observers exist is finite and small. To be precise, we define the existence of observers to be equivalent to the generation of entropy production. In addition, we focus this discussion on regular observers (as opposed to BBs) since they (we) are known to exist.

As emphasized above, previous proposals invoking the criterion of environmental selection have been concerned only with the conditions that allow for the nucleation of observers, without including the termination of the observers in the analysis. This selection has been done by either using static measures on some spatial hypersurface (which leads to ambiguous procedures for 'time' slicing), or by assigning to each pocket universe a statistical weight that is further weighted with the nucleation density of observers in each pocket. The weights are generally distributed with respect to the nucleation density. Independent of other pathologies (e.g., ambiguities in defining a global versus local description of measure, ratio of infinities, the dependence on the choice of observers, and definitions of the time parameter), these proposals share the property that the evolution and the decay rate of observers are not taken into account when constructing a selection criterion.

The motivation for ignoring the influence of the long term fate of observers-specifically their decay rate- on predicting $\Lambda$ has been based on the fact that once structure forms than it decouples from the Hubble flow. On this basis, it was expected that $\Lambda$ would have no influence on the evolution of the stars or observers after the universe reaches its DS phase.

Ignoring the influence of $\Lambda$ on the existence of observers is not correct for the DS spaces due to the eternally constant and finite values of its temperature $T_{D S}$ and its entropy $S_{D S}$ : The number of observers produced before the DS epoch decreases exponentially due to the superluminal expansion as they get expelled from causal contact, or equivalently since every co-moving observer sees an effective shrinking horizon.

As is well-known [2], in about a Hubble time the physical radius of our universe approaches the constant value $H^{-1} \simeq \Lambda^{-1 / 2}$ and our universe approaches thermal equilibrium from that time onward. As a result, the universe eventually maintains a constant temperature $T_{D S} \simeq H^{-1}$ at all points and a constant finite entropy $S_{D S} \simeq 3 / \Lambda$ for all (sufficiently late) times. After such an equilibrium is reached, the universe loses its ability to do physical work, and no heat engine can operate because the energy available is finite and is distributed over an infinite time interval. The universe and any observers it contains thus suffer from one kind of cosmological heat death. This behavior is a manifestation of the well-known information loss puzzle for DS states, since the finite constant value of the temperature and entropy leading to this cosmological heat death of observers are a consequence of the fact that the de Sitter horizon does not evaporate away (unlike black holes). If the horizon could evaporate away, then the temperature and the entropy of the DS space would not maintain their constant value, and this type of heat death would not occur.

Cosmological heat death [4] is the modern counterpart to classical heat death [25], which, roughly speaking, would take place if the universe reached a thermodynamic equilibrium. In such a state, the universe would have the same temperature at all points in space, so that no heat engine could operate and no work could be done. The expansion of the universe changes the concept of heat death as follows: for universes that are not vacuum dominated, the background 
temperature of the universe is continually decreasing, so that the universe can never reach the constant temperature state of classical heat death. Nonetheless, such a universe can become purely adiabatic, so that no more work can be done within it, and can thus suffer one type of cosmological heat death [4]; however, since there are no bounds on the entropy, observers can be continually produced via the flow of density perturbations accross the horizon, i.e., the universe need not become adiabatic. A DS universe, in contrast, can eventually reach a constant temperature state (at the DS temperature), and can thus suffer a cosmological heat death of another type. This second kind of cosmological heat death is the one relevant for investigating anthropic arguments for $\Lambda$ that consider observers at arbitrary cosmic epochs (as discussed here).

As argued above, any remnant observers in a DS universe end up in a cosmological heat death (of the second kind). Due to the constant finite entropy $S_{D S}$, no new entropy and hence no new observers can be generated. For these reasons, the universe in its DS epoch has no production channels for observers or entropy over an infinite time interval, while all the pre-DS epoch observers disappear within a short finite time. On the largest spatial scales, the cosmological heat bath shuts down entropy production on a time scale $\delta \tau \simeq \Lambda^{-2 / 3}$. On smaller scales, extant astrophysical objects (e.g., stars and stellar remnants) continue to generate energy and entropy until their fuel sources are exhausted. Although these time scales are somewhat longer than that of the cosmological heat bath [4], the lifetimes of these bodies are nonetheless finite.

It is straightforward to quantify this statement concerning the influence of $\Lambda$ on the number of observers. Let us denote by $\mathcal{N}=n a^{3}$ the number of observers in the Hubble volume, where $n$ is the number density and $a(\tau)$ is the scale factor, with $\tau=\int \mathrm{d} t / a$ the conformal time and $t$ physical time. The (co-moving) 3 -volume is given by $V_{3}=(4 \pi / 3)\left[\tau_{*}-\tau\right]^{3}$, where $\tau_{*}$ is the future asymptotic value of the conformal time in our causal domain. The 3 -volume $V_{3}$ thus approaches zero in the limit $t \rightarrow \infty\left(\tau \rightarrow \tau_{*}\right)$. This behavior represents the 'shrinking of the horizon' in co-moving coordinates. On the other hand, the (co-moving) 4-volume $V_{4}=\int V_{3} \mathrm{~d} t \propto H^{-2}$ has a finite value determined by the physical radius of the Hubble volume $H^{-1}$. The total number of observers in the co-moving volume is roughly proportional to the volume $\mathcal{N} \simeq V_{3}$, so that it decreases with the 'shrinking of the horizon' of $V_{3}$. The number of observers thus gets smaller according to

$$
\frac{\mathrm{d} \mathcal{N}}{\mathrm{d} t} \simeq \frac{\mathrm{d} V_{3}}{\mathrm{~d} t}=3 \mathcal{N} \frac{1}{\tau-\tau_{*}} \frac{\mathrm{d} \tau}{\mathrm{d} t}+\Gamma \mathcal{N} .
$$

Next we can define the parameter $x$ through

$$
x \equiv-\frac{1}{\tau-\tau_{*}} \frac{\mathrm{d} \tau}{\mathrm{d} t} \propto-\frac{\dot{V}_{3}}{3 \dot{V}_{4}},
$$

and find that the balance equation is

$$
\frac{\mathrm{d} V_{3}}{\mathrm{~d} t}=-3 x V_{3}
$$

Note that $x>0$ since $\mathrm{d} \tau / \mathrm{d} t$ is negative, and it varies between $H / 2$ in the past and 0 at future infinity. We have added a particle production $(\Gamma>0)$ or annihilation $(\Gamma<0)$ term for the most general case. If continued generation of entropy production were possible, then the above equation for the number rate would be modified by a source term $S(t)$ so that $\mathrm{d} \mathcal{N} / \mathrm{d} t \simeq \mathrm{d} V_{3} / \mathrm{d} t+S(t)$. But since the continued generation of entropy over the whole infinite time interval is strictly zero for DS spaces after reaching thermal equilibrium, we have $S(t)=0$. If $\Lambda$ is not strictly a cosmological constant, but rather a dynamic dark energy component, then quite possibly $S(t) \neq 0$ would contribute to the generation of observers. 
Ignoring $S(t)$ we can equivalently write this differential equation, as a balance equation in terms of the number density of observers $\mathrm{d} n / \mathrm{d} t+3 H n=-3 x n+\Gamma n$. Due to the cosmological heat death and thermal equilibrium in the Hubble volume $\Gamma \equiv 0$. Solving the differential equation we find that $\mathcal{N}=n a^{3}=\mathcal{N}_{0} e^{-3 x t}$ which reflects nothing more than the dilution of all objects expelled from our Hubble volume due to the accelerated expansion.

In short, we have shown that the number of observers $\left\langle\mathcal{N}_{j}\right\rangle=f_{j}$, crucial for the anthropic predictions for the distribution of the values of $\Lambda$ in $j$ bubbles, approaches zero in any space with a nonzero vacuum energy $\Lambda$ over an infinite time interval. Observers exist for a brief time interval around the $\Lambda$ domination era. By applying the Copernican time principle for the past, present, and future of our universe [4], which states that there is nothing special about our present epoch for picking this time to make a measurement, then making a measurement randomly in space and time in a de Sitter universe $j$ should yield an identically zero number of observers $f_{j}=\left\langle\mathcal{N}_{j}\right\rangle=0$. Constraining ourselves to the selection of a special timeslice when observers are known to exist, instead of a random one for making a measurement, than counts as fine-tuning rather than a prediction of the theory.

Although in this paper we are not concerned with the viability of eternal inflation, it should be noted that our argument for the cosmic heat death of observers can be extended to the case of the eternal inflation for the following two reasons: (i) the anthropic approach to the probability of the eternal inflation is given by the product of the number of bubbles with the number of observers in them, as reviewed above. Although our argument has nothing new to say about the number of bubbles produced, it does yield a vanishing number of observers in each bubble, thereby making the anthropic probability given by the product equal to zero; (ii) the global boundary condition we consider for this approach considers a timeslice, taken at time infinity. Since the parameter of time is conventionally taken to be in common for all the bubbles produced by eternal inflation then the timeslice in the far future would cut through all the bubbles. Since observers come into existence for a very brief time during the history of the bubble, then in this hypersurface at future infinity, the number of bubbles empty of observers will be overwhelmingly larger than the bubbles where observers are accidentally found.

We thus conclude that anthropic considerations (without additional assumptions, e.g., a preferred measurement time) are not consistent with the observed value of $\Lambda$. As a result, such arguments cannot be invoked as a means of predicting values of $\Lambda$ different from zero since the observers, which are at the heart of the anthropic principle, do not exist in the cold empty equilibrium DS spaces.

\section{An alternate argument allowing for large vacuum energy density}

As illustrated by the previous section, the basic point of this paper is that anthropic 'predictions' depend on what is assumed as a starting point (the 'priors' in the language of statistics). The above argument (section 3) allows for observers to be considered at any time, and then finds that the existence of observers essentially requires a zero value for the cosmological constant $\Lambda$. In this section for completeness we review the case when $\Lambda$ is not strictly a cosmological constant but a dynamic energy component, in which case the entropy bounds of DS spaces would not be applicable. Here we require that the universe produce observers before the present time (as in [3]), but allow the redshift of structure formation to change, and find that the value of $\Lambda$ can be millions of times larger than the observed value. A dynamic $\Lambda$ case has also been discussed in [14]) with similar conclusions to the ones here.

We note that the higher redshifts for structure formation considered here would require correspondingly larger density fluctuations in the early universe and hence larger 
temperature fluctuations in the cosmic background radiation. These alternate universes would not share all of the properties of our universe-only the requirement that observers can be produced.

For completeness we also note that the previous argument of section 3 applies specifically to the case in which $\Lambda$ is strictly constant. The situation may change if the dark energy density is time dependent, since there are no bounds on the entropy increase in this case, and hence the generation of observers may not be forbidden at randomly selected times for making a measurement. However, the argument constructed in this section applies to both the case of a cosmological constant and a dynamic dark energy component.

Specifically, the arguments of [3] start with the idea that large values of the vacuum energy $\rho_{V}$ (or, equivalently, $\Lambda=8 \pi G \rho_{V}$ ) suppress galaxy formation. As a result, in order to form nonlinear structures, the vacuum energy density must obey the bound

$$
\rho_{V}<\frac{500}{729} \tilde{\rho}
$$

where $\tilde{\rho}$ provides a measure of the starting size of the density perturbations $\Delta \rho$, i.e.,

$$
\widetilde{\rho} \equiv \lim _{t \rightarrow 0}\left\{\frac{[\Delta \rho(t)]^{3}}{\rho^{2}(t)}\right\} .
$$

After further algebra [3], one finds that the formation of structure (galaxies or quasars) before a redshift $z_{*}$ provides a lower bound on the maximum value of the parameter $\tilde{\rho}$ defined above:

$$
\left.\tilde{\rho}\right|_{\max } \geqslant \frac{729}{1500} \pi^{2} \rho_{0}\left(1+z_{*}\right)^{3},
$$

where $\rho_{0}$ is the present-day density of the universe.

The original argument invoked the fact that our universe is observed to have produced quasars before a redshift of $z_{*} \sim 4$.5. Using this value in equation (4.3), in conjunction with equation (4.1), implies that the anthropic upper bound on the vacuum energy density is at least as large as $\sim 550 \rho_{0}$. However, this version of the argument relies on the choice of $z_{*} \sim 4.5$ in order to find a numerical value (see also [14]). In the vast ensemble of possible universes, other regions of spacetime could form quasars earlier. Thus, one could consider larger values of the formation redshift. If we use $z_{*} \sim 100$, for example, then the bound gets weaker, so that we would only conclude that $\rho_{V}$ is less than $\sim 5000000 \rho_{0}$, a considerably weaker result.

Relaxing the redshift requirement for quasars thus allows for a much larger vacuum energy density than the original anthropic argument of [3], and a much larger value than we observe. One can even take this argument to its limit, and consider universes in which structure forms right after decoupling, which correspond to a redshift of $z_{*} \sim 1000$, so that the resulting bound becomes $\rho_{V}<10^{9} \rho_{0}$.

Note that this argument applies equally well to approaches that rely on the PS formalism. The PS formula is an error function that peaks around $10^{2} \rho_{*}$, which is the matter energy density at the time of matter-radiation equality. The special scale built into this formula is thus the redshift when the matter radiation equality occurs. We can easily envision, in the vast multitude spacetime regions in the multiverse, universes with different matter inventories, so that the matter-radiation equality occurs at very different redshifts from our universe. In this case, the probability described by the PS formula peaks at different values since the scale $\rho_{*}$ varies. As a result, the predicted value of $\Lambda$ (as a function of $\rho_{*}$ ) peaks at different values than in the case of our universe.

To summarize, we have argued that anthropic arguments with one set of assumptions (section 3, where observers are considered at all cosmic times) imply that $\Lambda=0$, whereas another set of assumptions (here, where cosmic structure can form earlier) predicts, or at least 
allows, $\Lambda$ to be a billion times larger than its observed value. The predictions of this class of anthropic arguments thus depend sensitively on the starting assumptions. This sensitivity, coupled with the lack of a way to specify a priori what the 'correct' assumptions should be, severely limits the predictive power of anthropic reasoning. As a result, the anthropic approach becomes an unfalsifiable theory.

\section{Conclusions}

This paper has investigated the viability of anthropic reasoning for predicting the value $\Lambda$ of the vacuum energy density of the universe. This discussion differs from previous considerations by incorporating the boundary conditions of the observers in eternally DS spaces, in particular, the fact that a finite amount of entropy is spread over an infinite time interval. Since entropy production is necessary for the continued existence of observers, the inability of universes with $\Lambda \neq 0$ to continue entropy production ultimately leads to the lack of observers. Specifically, we showed that the observers in any eternal de Sitter universe $(\Lambda \neq 0)$ suffer a cosmological heat death in the long term future. This finding, together with the fact that no continued entropy production is allowed, implies that the number of observers found by any given random measurement would be zero. Because of this lack of observers in DS spaces, anthropic approaches imply values of the vacuum energy density $\Lambda$ that are strictly zero with an overwhelmingly larger probability, proportional to the ratio of the 3 -volume over the 4-volume, a prediction that is not consistent with current observations of our universe.

In contrast to previous assumptions, $\Lambda$ has a significant influence on the existence of observers, even though nonlinear structures (halos) separate from the Hubble flow. Because the accelerating universe effectively separates (present-day) dark matter halos into island universes in the future [23], each such bound structure has finite energy and entropy resources, and hence a decaying number of observers. Any remaining observers also suffer a cosmological heat death. Thus, because of the vacuum energy, random measurements performed at any randomly picked time would typically find the number of observers in spaces with nonzero vacuum energy to be zero. In this sense, anthropic considerations do not predict the observed value of $\Lambda \neq 0$. For completeness, we note that these considerations do not apply for open universes; unlike the spatially flat DS space with a fixed physical radius $H^{-1}$, open universes lack a spatial bound and have no limit for their entropy and temperature.

This severe measure problem lies at the heart of the information loss puzzle for de Sitter universes. Taking into account the eternal nature of de Sitter space ${ }^{3}$, in combination with the fact that DS space soon empties out of observers and all its matter content, leads to the conclusion that most observers nucleated before vacuum energy domination are soon after lost from causal contact; the ones that survive into the DS era then suffer a cosmological heat death. Furthermore, no net production of observers in the eternal DS epoch is allowed since their nucleation would contribute an entropy channel to the DS entropy. At all times the observers decay rate is at least as large as the nucleation rate in DS spaces. For example, in our universe protons decay with a time scale in the range $10^{33}-10^{45} \mathrm{yr}$, where the lower limit comes from current experimental bounds [24] and the upper limit is theoretical [4]. Similarly, black holes evaporate on a time scale roughly given by $\tau=10^{65} \mathrm{yr}\left(M_{\mathrm{bh}} / M_{\odot}\right)^{3}$, so that even the largest black holes are gone after $\sim 10^{92} \mathrm{yr}$; these objects provide the last important contributions to the background non-equilibrium processes for astrophysical remnants inside the causally

\footnotetext{
3 At least at the classical level [2] DS space is eternal with constant and finite temperature and entropy. This solution may change when quantum effects and back reactions on the DS geometry are taken into account [13, 15]. In the latter case, DS space may decay in astronomical scales due to instabilities from quantum effects.
} 
connected region [4]. From then to infinity the Hubble volume attains thermal equilibrium with the same temperature $T_{D S}$ at all points and time.

Using a Copernican principle approach, that is, randomly making measurements for observers in DS spaces, it then follows from above considerations that the probability to randomly find an observer at any time has a measure zero. This result is independent of how the time parameter is defined. This argument is, in essence, a different way of stating the well known problem of information loss, but it is applied here to anthropic considerations for predicting the typical values for $\Lambda$. As outlined above, anthropic selection thus requires $\Lambda$ to be strictly zero, in conflict with the observed value for our universe.

The difference between previous anthropic predictions and those of this paper can be summarized as follows: previous arguments assume that the only observers that should be considered are those in the present day universe, whereas this paper adopts a more global view, with observers being allowed to exist at any epoch. If we limit the possibilities from the onset to the case of finding observers in a young universe, $t<14 \mathrm{Gyr}$, then a small nonzero value of $\Lambda$ does not hurt the odds; however, if we consider the question of observers in the universe at any time, then any small nonzero value of $\Lambda$ is devastating. So the question of whether or not anthropic arguments imply, or even allow, nonzero values of $\Lambda$ depends on whether the present cosmological epoch is special or not. In other words, the predictions of anthropic arguments depend on whether or not one adopts the Copernican time principle. The predictions of anthropic arguments can be radically different for varying initial assumptions and thus their predictive power is severely limited.

\section{Acknowledgments}

We would like to thank Lee Smolin and Dragan Huterer for useful discussions, and the organizers of the $2007 \mathrm{fq}(\mathrm{x})$ conference, Max Tegmark and Anthony Aguirre, for helping to facilitate this collaboration. FCA is supported by the Michigan Center for Theoretical Physics and by the Foundational Questions Institute through grant RFP1-06-1. LM-H is supported in part by DOE grant DE-FG02-06ER1418 and NSF grant PHY-0553312.

\section{References}

[1] Reiss A G et al 1998 Astron. J. 1161009

Perlmutter S et al 1998 Astrophys. J. 517565

Garnovich P H et al 1998 Astrophys. J. $\mathbf{5 0 7} 74$

[2] Gibbons G W and Hawking S W 1977 Phys. Rev. D 152738

[3] Weinberg S 1987 Phys. Rev. Lett. 59

[4] Adams F C and Laughlin G 1997 Rev. Mod. Phys. 69337

[5] Vilenkin A 2007 J. High Energy Phys. JHEP01(2007)092 (Preprint hep-th/0611271) and references therein

[6] Easther R, Lim E A and Martin M R 2006 J. Cosmol. Astropart. Phys. JCAP03(2006)016

[7] Vilenkin A 2006 Preprint hep-th/0611271

[8] Mersini-Houghton L and Parker L 2007 Preprint arXiv:0705.0267 Aguirre A 2007 Preprint arXiv:0712.0571

[9] Bousso R, Harnik R, Kribs G D and Perez G 2007 Preprint hep-th/0702115

[10] Starkman G D and Trotta R 2006 Phys. Rev. Lett. 971301 Smolin L 2004 Preprint hep-th/0407213

[11] Tegmark M, Aguirre A, Rees M J and Wilczek F 2005 Preprint astro-ph/0511774

[12] Martel H, Shapiro P R and Weinberg S 1998 Astrophys. J. 49229

[13] Mersini-Houghton L 2006 Preprint gr-qc/0609006

[14] Aguirre A 2001 Phys. Rev. D 64083508 (Preprint astro-ph/0106143)

[15] Page D N 2006 Preprint hep-th/0612137

[16] Press W H and Schetcher P 1974 Astrophys. J. 187425 
[17] Guth A 2000 Phys. Rep. 333555

[18] Bousso R 2000 J. High Energy Phys. JHEP11(2000)038 (Preprint hep-th/0010252)

Bousso R, Maloney A and Strominger A 2002 Phys. Rev. D 65104039 (Preprint hep-th/0112218)

Goher N, Kleban M and Susskind L 2003 J. High Energy Phys. JHEP07(2003)056 (Preprint hep-th/0212209)

Kaloper N, Kleban M, Lawrence A, Shenker S and Susskind L 2002 J. High Energy Phys. JHEP11(2002)037 (Preprint hep-th/0209231)

[19] Dyson L, Kleban M and Susskind L 2002 J. High Energy Phys. JHEP10(2002)011 (Preprint hep-th/0208013)

[20] Albrecht A and Sorbo L 2004 Phys. Rev. D 70063528 (Preprint hep-th/0405270)

[21] Susskind L 2003 Preprint hep-th/0302219

Susskind L 2004 Preprint hep-th/0405189

[22] Halliwell J J and Hawking S W 1985 Phys. Rev. D 318

[23] Adams F C, Busha M T, Evrard A E and Wechsler R H 2003 Int. J. Mod. Phys. 121743

Krauss L M and Scherrer R J 2007 Gen. Rev. Grav. 391545

[24] Super-Kamiokande Collaboration 1999 Phys. Rev. Lett. 831529

[25] von Helmholz H 1854 On the Interactions of Natural Forces, reprinted in 1961 in Popular Scientific Lectures ed M Kline (New York: Dover)

Clausius R 1865 Ann. Phys. Lpz. 125353 